\title{
Using surveillance data for action: lessons learnt from the second generation HIV/AIDS surveillance project in Pakistan
}

\author{
F. Emmanuel, ${ }^{1}$ A. Adrien, ${ }^{2}$ U. Athar, ${ }^{1}$ M. Imran, ${ }^{3}$ T. Reza ${ }^{7}$ and J. Blanchard ${ }^{1}$
}

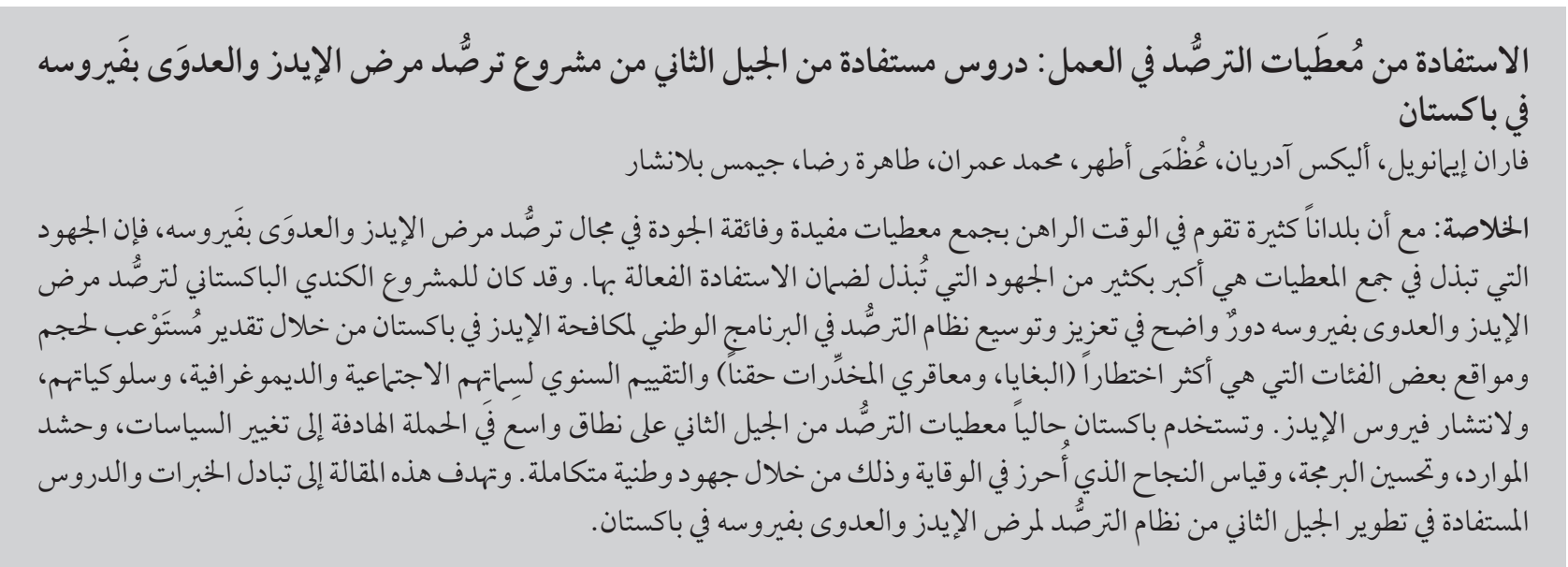

ABSTRACT Although many countries are now collecting useful, high-quality HIV surveillance data, more efforts are put into the collection of data than into ensuring it is deployed effectively. The Canada-Pakistan HIV/ AIDS surveillance project has strengthened and expanded the existing national AIDS control programme surveillance system in Pakistan through a comprehensive estimate of the size and location of some of the most at-risk populations (sex workers and injection drug users) and annual assessments of their sociodemographic characteristics, behaviours and HIV prevalence. The country now uses second generation surveillance data at a broad level to lobby for policy change, mobilize resources, improve programming and measure the success of prevention through an integrated national effort. This article aims to share the experiences and lessons learnt in the development of a second generation surveillance system for HIV/AIDS in Pakistan.

Utilisation des données de surveillance pour l'adoption de mesures : enseignements tirés du projet de surveillance de deuxième génération du VIH/sida au Pakistan

RÉSUMÉ De nombreux pays collectent des données de surveillance utiles et de haute qualité sur le VIH. Toutefois, leurs efforts sont davantage axés sur le recueil des données que sur une exploitation efficace. Le projet de surveillance du VIH/sida Canada-Pakistan a renforcé puis étendu le système de surveillance du programme national de lutte contre le sida existant au Pakistan, au moyen d'une estimation globale de la taille et de la distribution géographique de certains groupes à plus haut risque (travailleurs du sexe et consommateurs de drogues injectables) et d'évaluations annuelles de leurs caractéristiques sociodémographiques, de leurs comportements et de la prévalence du VIH. Le pays a désormais recours à des données de surveillance de deuxième génération à grande échelle pour faire pression en faveur de changements politiques, de la mobilisation de ressources, de l'amélioration des programmes et du suivi de la réussite de la prévention par une action nationale coordonnée. Le présent article vise à diffuser les données d'expérience et les enseignements tirés de l'élaboration du système de surveillance de deuxième génération du VIH/sida au Pakistan. 


\section{Introduction}

The concept of second generation surveillance (SGS) for HIV/AIDS as the "regular, systematic collection, analysis and interpretation of information for use in tracking and describing changes in the HIV/AIDS epidemic over time" was first introduced by the World Health Organization and Joint United Nations Programme on HIV/AIDS. Since then, a number of countries have tailored it to their specific needs in order to understand and describe the stages of their HIV epidemic [1-3]. Although many countries are now collecting useful, high-quality data, it has been noted that more efforts are put into the collection of data than into ensuring it is deployed effectively [4-6].

Surveillance systems enable a country to understand the magnitude of the epidemic and monitor trends over time [7]. Surveillance is not a onetime activity, however, nor should it be a stand-alone programme. Information about trends in HIV prevalence, which populations are affected, and so on, can help a country to monitor its epidemic and also provide information about the effectiveness of prevention and control measures. Estimating the numbers and mapping the geographical location of groups at high risk of contracting and spreading HIV within a given community are fundamental to ascertaining the scale of required interventions and identifying areas where prevention efforts need to be focused. Behavioural data, for example, can serve as an early warning system that immediate action is needed and help in the design of effective services and programmes [8]. Furthermore, tracking risk behaviours over time enables the effectiveness of intervention programmes to be monitored [9]. These data can also be used to strengthen the commitment of communities and mobilize them towards a more effective response [10,11].

This article aims to share the experiences and lessons learnt in the development of an SGS system for HIV/AIDS in Pakistan. In addition, we document the use of SGS data for advocacy, ensuring commitment from policy-makers and developing a national response for combating HIV/ AIDS in Pakistan.

\section{Second generation surveillance in Pakistan}

Apart from other interventions, surveillance has always remained a major focus of the national response to HIV/ AIDS in Pakistan. The surveillance systems in place largely relied on the collection, analysis and reporting of data from routinely reported diagnoses of notifiable diseases, based either on clinical or laboratory diagnoses. HIV serosurveillance, however, is of limited use in places where HIV infection is relatively uncommon among the general population. SGS for HIV/AIDS not only captures the seroprevalence trends but also the underlying risk behaviours which actually determine the course of epidemic in a given population [1].

In 2003, the Pakistan national AIDS control programme expanded its response to the epidemic via an enhanced HIV/AIDS control programme [12]. As a part of this response, the Canadian International Development Agency provided assistance for the establishment of an effective national SGS system for HIV/AIDS through the Canada-Pakistan HIV/AIDS Surveillance Project (HASP). This was implemented by a consortium of Agriteam Canada Consulting Ltd, the University of Manitoba and ProAction: Partners for Community Health Inc., with technical assistance from the Public Health Agency of Canada.

Since 2004, HASP has led to the expansion of the surveillance and monitoring system for HIV/AIDS in Pakistan to focus on most at-risk populations (MARPs). A key component of this is a comprehensive estimation of the numbers of MARPs and the HIV prevalence among subgroups, as well as describing some of their sociodemographic characteristics and behaviours. This is now conducted in all major cities of Pakistan on a yearly basis. The MARPs surveyed include 4 target groups: female sex workers (FSWs), male sex workers (MSWs), hijra sex workers (HSWs) and injection drug users (IDUs). HSWs are transgender individuals who form a unique type of sex worker in Pakistan and are of 2 major types, khusras and zananas.

Since implementation of the SGS in 2004 and after pilot testing the methods, Pakistan has conducted 3 annual rounds of surveillance with IDUs, MSWs and HSWs and collected data in 8 major cities in 2005-06, which were increased to 12 in 2006-07 and another 9 in 2007-08 [13-15]. For FSWs 2 rounds of surveillance were conducted only in 2005-06 and 2006-07.

\section{Surveillance methods}

\section{Mapping data}

Each year, data collection was done in 2 phases [16]. Phase 1 consisted of a rapid, geographic mapping to estimate the numbers, location and typologies of the 4 MARPs (FSWs, MSWs, HSWs and IDUs) in major cities across all 4 provinces. The mapping methodology consisted of dividing the cities into geographical zones and within each zone interviewing key informants to obtain information about the location and size of each risk population.

The mapping data were entered into a database specifically designed for the study. To generate final estimates of each risk population, the estimate ranges for each site and location were rolled up, first for a zone and then for the city, to produce minimum and maximum estimates. The mean of the minimum and maximum estimates was used as a single best estimate. 


\section{Behavioural and biological survey}

Information collected in phase 1 was verified in phase 2 with a cross-sectional behavioural and biological survey using interviews with members of all 4 target groups [16]. The mapping data provided sampling frames, and diverse sampling techniques were used to draw representative samples of the target populations in each city. A sample size of 400 was set for each risk population in every city, based on assumptions about baseline HIV prevalence, expected changes in prevalence and key behavioural characteristics of the study population.

Behavioural data were collected by trained interviewers using structured questionnaires that were designed in English and subsequently translated into the local language. These included questions on sociodemographic and personal characteristics, as well as a core set of risk behaviour indicators to monitor behaviour patterns in the key populations. Informed consent was obtained prior to conducting the interviews. Biological data were gathered using the capillary dried blood-spot method, chosen for its ease of collection, storage, shipping and serological accuracy [17]. The interviewers were trained in dried blood-spot collection and infection control processes. A debriefing session was held with the interviewees on completion of the questionnaire and blood sample, to answer any queries and provide information about HIV prevention and the services available including voluntary counselling and testing services.

A total number of 9450 IDUs, 5275 MSWs and 4898 HSWs were interviewed during the 3 surveillance rounds of 2005-08, while 7773 FSWs were surveyed in the 2 rounds of 2005-07.

The behavioural and biological data were stored and analysed at the central data coordinating unit of the national AIDS control programme in Islamabad. Data entry was done using computer software developed for the study. The behavioural database was linked with the laboratory results by an encrypted unique identifier and unique study site code. The final analysis was done using SPSS, version 12.0.

\section{Outcomes}

\section{Population size estimates and operational dynamics}

Results of the mapping studies conducted in the 12 cities of Pakistan are shown in Table 1. The largest group of MARPs (54.7\%) were FSWs, with an estimated number of 79127 . Based on information on group dynamics FSWs were categorized into various typologies. The largest typology identified was home-based FSWs: sex workers who live at home with their families and are involved in covert sexual activities through contacts with pimps, madams/ aunties and/or network operators. Street-based FSWs, the second largest typology identified, solicit clients in public places through cruising sites or pick-up points. Kothikhana-based FSWs live in small premises/houses rented by a madam and/or a network operator and entertain clients there. The traditional brothel-based sex workers comprised only $1.2 \%$ of the overall female sex workers. In addition to these categories, a small number of FSWs (1.2\%) were reported to operate as call girls and hotel-based sex workers.

\begin{tabular}{|c|c|c|}
\hline Most at-risk population & Mean no. & Range \\
\hline \multicolumn{3}{|l|}{ Female sex workers } \\
\hline Total & 79127 & $67258-90996$ \\
\hline Home-based & 37925 & $31556-42694$ \\
\hline Kothikhana-based & 16125 & 15 491-20 958 \\
\hline Street-based & 23498 & $19123-25872$ \\
\hline Brothel-based & 950 & 807-1092 \\
\hline Other (call girls, hotel-based) & 979 & 832-1126 \\
\hline Average per cruising site & 5.7 & $3-31$ \\
\hline \multicolumn{3}{|l|}{ Injection drug users } \\
\hline Total & 31555 & $24496-36213$ \\
\hline Average per site & 7.5 & $2-12$ \\
\hline \multicolumn{3}{|l|}{ Hijra sex workers } \\
\hline Total & 14725 & 12 517-16 933 \\
\hline Average per cruising site & 5.2 & $3-7$ \\
\hline \multicolumn{3}{|l|}{ Male sex workers } \\
\hline Total & 19320 & $16422-22218$ \\
\hline Average per site & 4.7 & $2-11$ \\
\hline
\end{tabular}


IDUs formed the second largest group of MARPs (21.8\%), with an estimated number of 31555 spread over 4199 sites. Two major types of IDUs were identified - street-based or homebased - depending on the place where the risk activity took place.

MSWs comprised of $13.3 \%$ of all MARPs (estimated total number of 19320).

HSWs accounted for $10.2 \%$ of all MARPs (estimated total number of 14725).

\section{HIV seroprevalence}

Figure 1 shows the trend of HIV infection rate among all MARPs surveyed in Pakistan over 2005-08. This shows that the highest rate of HIV infection was among IDUs and that infection rates among IDUs have been increasing, from $10.8 \%$ in 2005 to $20.8 \%$ in 2008. Not only has the overall prevalence increased, but the number of sites with relatively advanced epidemics has also expanded. All 8 cities where surveys were conducted in 2008 showed prevalence rates of over 5\% among IDUs, in comparison with 5 out of 8 cities in 2005 and 8 out of 12 cities in 2006-07 (data not shown). Although IDUs have the highest infection rates, $\mathrm{HIV}$ is beginning to become established in other MARPs such as HSWs, for whom the overall HIV prevalence rose to $6.4 \%$ (95\% CI: $5.0 \%, 7.7 \%$ ) in 2008 (Figure 1). Substantial rates of infection are now reported among HSWs in several cities; the highest prevalence was reported in Larkana (27.6\%) whereas all other cities had less than $5 \%$ prevalence (data not shown). The infection rate among MSWs in 2008 was unchanged from 2006 , i.e. $0.9 \%$ ( $95 \%$ CI: $0.3 \%$, 1.5\%). Although FSWs formed the largest MARP in terms of numbers, only 1 FSW was found to be HIV positive in 2007, in comparison with 3 who tested positive in 2006.

\section{Behavioural characteristics}

Table 2 shows the trends in high-risk sexual and injecting behaviours among MARPs in Pakistan. The average numbers of clients for all the 3 groups of sex workers remained between 2-3 clients per day, with little variation across the years. Consistent condom use over the past month with paying clients was significantly improved for MSWs (from $6.8 \%$ to $24.0 \%, P<0.001)$, HSWs $(5.8 \%$ versus $19.7 \%, P<0.001)$ and FSWs (from $18.0 \%$ to $23.0 \%$ ). Condom use at the last commercial sexual encounter also significantly increased among all MARPs over time. Among IDUs, risky practices have been decreasing. Sharing of needle/syringes at the last injection significantly decreased from $47.1 \%$ in 2005 to $17.6 \%$ in $2008(P<0.001)$, along with reuse of syringes, which also declined from $35.2 \%$ in 2005 to $22.5 \%$ in $2008(P<0.001)$. However, there was evidence of IDUs having sex with FSWs in the previous 6 months. Behavioural data also suggested that the potential for the epidemic to spread among MSWs is high.

The utilization of services provided by the various service delivery programmes for MARPs has increased over

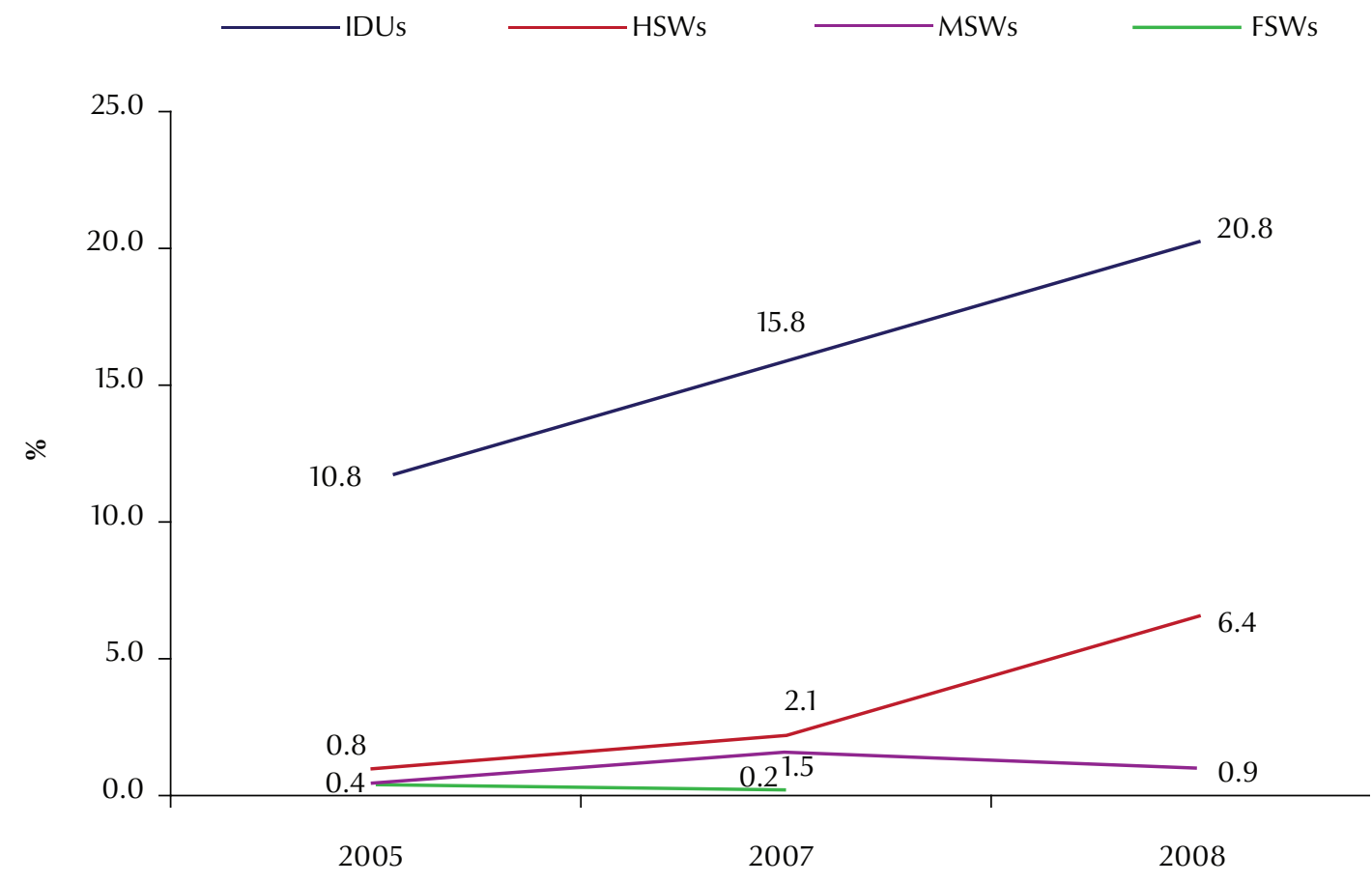

Figure 1 Emerging HIV infection rates among most at-risk populations in Pakistan, 2005-08 (IDUs = injection drug users; HSWs = hijra sex workers; MSWs = male sex workers; FSWs = female sex workers) 


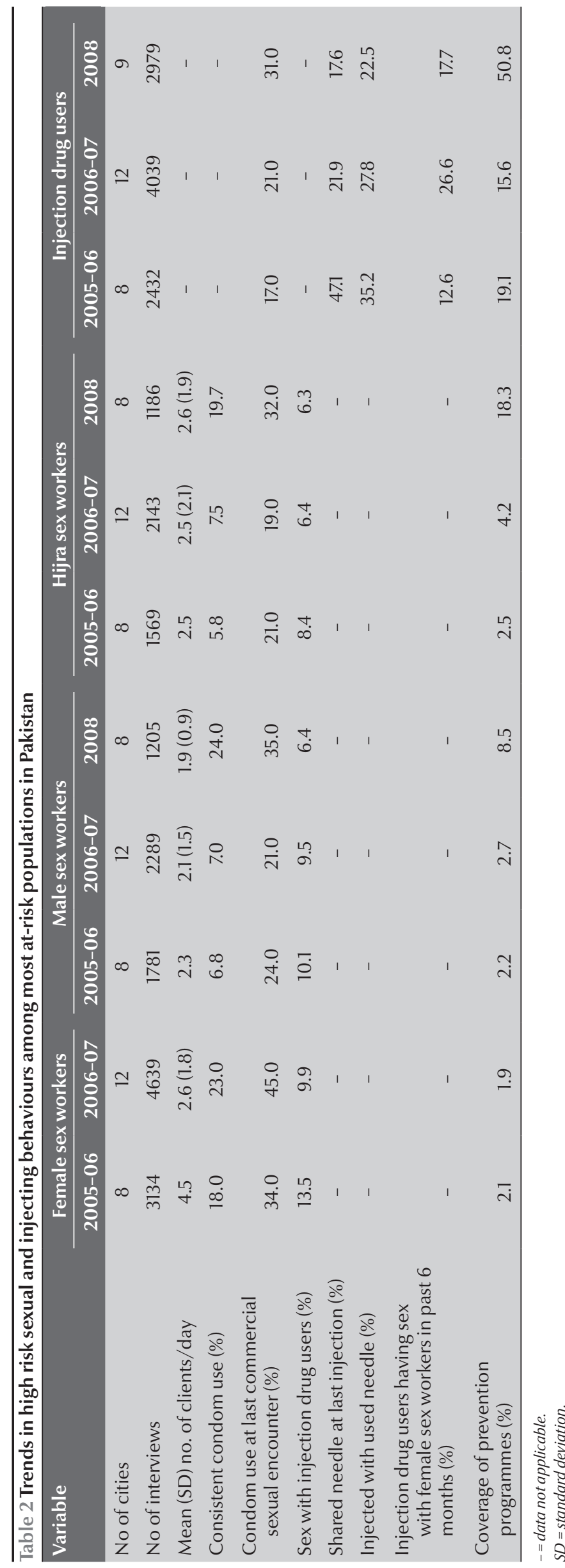

time. Among MSWs service utilization increased to $8.5 \%$ in 2008 from $2.2 \%$ in 2005 , while for HSWs it increased from $2.5 \%$ in 2005 to $18.3 \%$ in 2008. Likewise the utilization of services showed a significant improvement for IDUs from 19\% in 2005 to $51 \%$ in $2008(P<0.001)$. However no significant change was observed for FSWs.

\section{Utilizing the data}

\section{Defining the HIV epidemic and estimating the size of MARPs}

The surveillance data collected over the years has highlighted the changing patterns of HIV in Pakistan. Mapping studies of MARPs have provided size estimates of each of the populations exposed within each city surveyed. Serial HIV seroprevalence data has shown that like many other countries in south Asia, Pakistan's HIV epidemic is concentrated among IDUs throughout the country and that infection rates among IDUs are increasing. Prevalence trends suggest that HIV is beginning to become established in other MARPs as well. Substantial levels of infection are now reported among transgender sex workers in several cities [15]. In addition to these index populations, the estimates of prevalence for other populations at risk (sexual partners of IDUs, clients of sex workers) were derived from a combination of HIV data from these index groups and behavioural data that measured the level of exposure to the index group.

\section{Policy development and advocacy}

Once the magnitude of the HIV burden had been determined, this information was used to develop the first national HIV/AIDS policy. It highlighted the need for a coordinated, comprehensive, and multisectoralHIV prevention and control response in Pakistan. Provision of harm-reduction services to various high-risk groups at a scale necessary to halt the establishment of HIV among these groups and avoid its spread to the general population was acknowledged as a prime objective of the national response. For a long time, the existence of a sizeable commercial sex industry in Pakistan was denied by the government authorities. The mapping data was used as an advocacy tool to policy-makers and religious leaders to demonstrate the existence of large concentrations of high-risk groups and the consequences of the unfolding HIV epidemic. For the first time new interventions, e.g. 
opioid substitution therapy, were recommended for inclusion in the national HIV prevention and control response. HIV prevalence trend data were used to sensitize the media and other relevant bodies to ensure their involvement in scaling-up the national response [18].

\section{Development of an integrated national response and resource allocation}

SGS data provided valuable information for developing the second national HIV/AIDS strategic framework through which all donors and stakeholders were brought together to define a single national HIV prevention and control agenda and set priorities for the allocation of resources for the next 5 years. Based on the HIV scenario highlighted by the SGS data, a proposal to the Global Fund to Fight AIDS, Tuberculosis and Malaria was developed for round 9, clearly focussing on providing services for IDUs, who are currently the hub of HIV infection in the country. At the national level, estimates of the size of various high-risk groups have helped determine the universal access targets as well as set targets in line with the Millennium Development Goals. Various other programme proposals developed within this national HIV response have followed an evidencebased approach, allocating all available resources where the burden of infection is the highest.

\section{Designing and scaling up services for MARPs}

Before SGS was effective in Pakistan, interventions among high-risk groups were programmed with limited knowledge about the numbers, typology and geographical distribution of these groups. The establishment of the SGS system as a core programme component provided context-specific epidemiological evidence to guide programme development and implementation at all levels. The behavioural data were used to develop standards of service delivery for each of these high-risk groups, and the biological data were used to channel resources to the groups and areas most affected by the epidemic. The data were also utilized by various organizations for developing and refining service delivery programmes to these high-risk groups. For example, SGS data collected on the use of sterile injection equipment by IDUs led to the expansion of the needle exchange programme for IDUs in Punjab [19]. Likewise, the recognition of high-risk anal sex among men who have sex with men led to the distribution of water-based lubricants along with condoms by the outreach staff of various service delivery programmes.

At provincial level a number of new cities were included in the programmes, based on the mapping information available from SGS. Thus, geographical data helped in planning service delivery sites, while population estimates helped to determine the number and extent of services required for appropriate coverage, programme staffing needs, service infrastructure and commodities to be provided. The information on the subtypes and operational dynamics within each MARP was used to design interventions and determine modes of service delivery. For example, outreach strategies were planned differently for brothel-based FSWs than for homebased FSWs.

\section{Using data for evaluating programme success}

Although programme evaluation and surveillance activities overlap, they are not the same and they serve different purposes. However, we used SGS data to determine whether or not prevention andcare programmeshave beensuccessful and are able to control and contain emerging epidemics. With SGS data, the estimates of the number of MARPs provided the denominators to make basic calculations about monitoring indicators and set baselines for key outcomes and indicators. Thus, improved levels of HIV knowledge, consistent condom use, reduction in sharing of needle/syringes, improved programme coverage and use of available services by target populations, all served as markers for programme success. The combined analysis of these datasets along with data collected by the services provides a valid assessment of the effects of service delivery programmes on the course of the epidemic.

\section{Conclusions and lessons learnt}

The SGS system in Pakistan has provided a comprehensive response to the data needs of the country. In addition to understanding the magnitude of the epidemic and monitoring its trend, SGS data in Pakistan has been used extensively by the national and provincial programmes to lobby for policy change, mobilize resources, improve programming and measure the success of prevention through an integrated national response.

A number of lessons have been learned from this initiative:

- Surveillance is not a stand-alone programme and has to be embedded in the overall national response to HIV.

- Surveillance is not primarily a research activity, but should be used to provide reliable documentation of trends in epidemic prevalence and associated risk behaviours,

- Mapping is an integral part of planning HIV response. Knowledge about population estimates, locations and the group dynamics of MARPs is the cornerstone of planning services for these groups,

- Simpler forms of analyses are useful. An evaluation of different risk behaviours among various subpopulations through simple descriptive analysis is very valuable for steering prevention activities. Likewise, a description of HIV prevalence levels is needed to 
show where prevention efforts need to be concentrated.

- Finally, we have learnt that evidence provided by surveillance data plays a strong role in advocacy. However, communication strategies should be developed to present data in a clear, effective way that is accessible to a wide audience. SGS data can be effectively used at a broad level to lobby for policy change, mobilize resources, improve program- ming and measure the success of prevention through an integrated national effort. Such effort needs to be continued for an improved national response to this emerging epidemic.

\section{References}

1. Second generation Surveillance for HIV/AIDS: the next decade. Geneva, World Health Organization/Joint United Nations Programme on HIV/AIDS, 1999.

2. Guidelines for Second generation HIV Surveillance. Geneva, World Health Organization/Joint United Nations Programme on HIV/AIDS, 2000

3. Meeting the behavioral data collection needs of national HIV/ AIDS and STD programmes. Arlington, Virginia, Family Health International/IMPACT and Joint United Nations Programme on HIV/AIDS, 1998.

4. Garcia-Calleja JM et al. A global analysis of trends in the quality of HIV sero- surveillance. Sexually Transmitted Infections, 2004, 80(Suppl. 1):i25-30.

5. Pisani E et al. HIV surveillance: a global perspective. Journal of Acquired Immune Deficiency Syndromes, 2003, 32(Suppl. 1):S3-11.

6. Lyerla R, Gouws E, Garcia-Calleja JM. The quality of serosurveillance in low- and middle income countries: status and trends through 2007. Sexually Transmitted Infections, 2008, 84 (Suppl. I):i85-91.

7. Brown T et al. Improving projections at the country level: the UNAIDS Estimation and Projection Package 2005. Sexually Transmitted Infections, 2006, 82 (Suppl. 3):iii34-40.

8. The BSS Development and Implementation Group. Behavioral surveillance surveys: guidelines for repeated behavioral surveys in populations at risk of HIV. Arlington, Virginia, Family Health International, 2000

9. Brown T. Behavioral Surveillance: current perspectives, and its role in catalyzing action. Journal of Acquired Immune Deficiency Syndromes, 2003, 32(Suppl. 1):S12-17.

10. Garnett GP et al. Maximizing the global use of HIV surveillance data through the development and sharing of analytical tools. Sexually Transmitted Infections, 2004, 80 (Suppl. I):i1-4.
11. Thomas R et al. Second-generation HIV surveillance: better data for decision-making. Bulletin of the World Health Organization, 2004, 82:121-127.

12. National AIDS Control Programme. National strategic framework. Islamabad, Pakistan, Ministry of Health, 2002.

13. National AIDS Control Program. HIV/AIDS surveillance project. Integrated biological and behavioral surveillance. Round 1 report: 2005. Islamabad, Pakistan, Ministry of Health, 2005.

14. National AIDS Control Program. HIV/AIDS surveillance project. Integrated biological and behavioral surveillance. Round 2 report: 2006-07. Islamabad, Pakistan, Ministry of Health, 2007.

15. National AIDS Control Program. HIV/AIDS surveillance project. Integrated biological and behavioral surveillance. Round $3 \mathrm{re-}$ port: 2008. Islamabad, Pakistan, Ministry of Health, 2008.

16. National AIDS Control Program. HIV/AIDS surveillance project. Mapping: field operations and monitoring manual. Islamabad, Pakistan, Ministry of Health, 2008.

17. Solomon SS et al. Dried blood spots (DBS): A valuable tool for HIV surveillance in developing/tropical countries. International Journal of STD \& AIDS, 2002, 13:25-28.

18. Programme for Research and Capacity Building in Sexual and Reproductive Health and HIV in Developing Countries. HIV in Pakistan: preventing a future epidemic in most-at-risk groups. London, London School of Hygiene and Tropical Medicine, 2009

19. Emmanuel F, Fatima M. Coverage to curb the emerging HIV epidemic among Injecting drug users in Pakistan: delivering prevention services where most needed. International Journal of Drug Policy, 2008, 19 (Suppl. 1):S59-64. 